\title{
Batch Process Optimization via Run-to-Run Constraints Adaptation
}

\author{
Alejandro Marchetti, Benoît Chachuat and Dominique Bonvin
}

\begin{abstract}
In the batch process industry, the available models carry a large amount of uncertainty and can seldom be used to directly optimize real processes. Several measurement-based optimization methods have been proposed to deal with model mismatch and process disturbances. Constraints often play a dominant role in the dynamic optimization of batch processes. In their presence, the optimal input profiles are characterized by a set of arcs, switching times and active path and terminal constraints. This paper presents a novel method tailored to those problems where the potential of optimization arises mainly from the correct set of path and terminal constraints being active. The input profiles are computed between successive runs by dynamic optimization of a fixed nominal model, and the constraints in the optimization problem are adapted using measured information from previous batches. Note that, unlike many existing optimization schemes, the measurements are not used to update the process model. Moreover, the proposed approach has the potential to uncover the optimal input structure. This is demonstrated on a simple semi-batch reactor example.
\end{abstract}

Keywords: Dynamic optimization, Batch processes, Measurement-based optimization, Run-to-run optimization.

\section{INTRODUCTION}

In batch processing, there is often a great potential for process improvement via dynamic optimization [1]. However, the implementation of dynamic optimization methodologies continues to be rather rare in industry. One of the reasons for this is the lack of accurate models, because such models can rarely be found with affordable effort [1]. For example, the stoichiometry and kinetics of reaction systems are often insufficiently characterized. Furthermore, since reactions are usually modeled at the laboratory scale, the description might be inaccurate for commercial-scale reactors due to differences in mass and heat transfer, residence-time distributions, surface-to-volume ratios, and heat-removal capabilities.

In the presence of uncertainty in the form of model mismatch and process disturbances, the open-loop implementation of off-line calculated optimal inputs leads to suboptimal operation. Worse, the satisfaction of safety constraints and product quality specifications is no longer guaranteed unless a "conservative" strategy is adopted, i.e., a strategy that guarantees constraint satisfaction even in the worst-case situation [2]. However, conservatism is detrimental to the optimization objective.

Batch processes are characterized by two types of outputs, the run-time outputs (quantities that can be measured on-line) and the run-end outputs (quantities that are only available at

A. Marchetti, B. Chachuat and B. Bonvin are with the Laboratoire d'Automatique, École Polytechnique Fédérale de Lausanne, CH-1015 Switzerland final time) [3]. Also, since batches are typically repeated, two independent 'time' variables are available, i.e., the run time $t$ and the run index $k$. On the other hand, dynamic optimization problems have two types of constraints: the path constraints limit the inputs and states during the batch, while the terminal constraints limit the outcome of the batch at final time. These characteristics of batch processes and dynamic optimization problems make it possible to devise run-to-run (also called batch-to-batch) optimization schemes. The idea is to get to the optimum within a few batches, by exploiting the knowledge from previous batches. This way, it is possible to account for uncertainties and disturbances that are repetitive in every batch. However, random disturbances within a batch cannot be accounted for.

The industrial need for optimization methods that do not rely on an accurate process model has resulted in the design of various measurement-based optimization (MBO) methods. The idea is to use measurements in order to reduce conservatism, thereby leading to improved performance. MBO methods can be classified according to whether or not a process model is used to guide the optimization [4].

In implicit optimization, measurements are used to directly update the inputs towards the optimum. In essence, a feedback law is sought that implicitly solves the optimization problem. Recently, Srinivasan and Bonvin [5] proposed to derive the feedback structure from the necessary conditions of optimality (NCO). This approach, referred to as NCO tracking, exploits the structure of the optimal solution (type and sequence of arcs and set of active path and terminal constraints), which is assumed to be known. The inputs calculated from a nominal model are first parameterized; then, each input arc or parameter is linked to a specific part of the NCO; finally, the NCO are satisfied by adjusting the input arcs and parameters using measurements. A limitation of NCO tracking, however, is that it assumes that the solution structure does not change in the presence of uncertainty.

In explicit optimization schemes, on the other hand, the inputs are updated via the repeated optimization of a process model. Typically, the available measurements are used for model refinement [2], [6], [7], which implies an iteration between identification and optimization (two-step approach). However, the optimal inputs often fail to provide sufficient excitation for estimating the uncertain parameters accurately. The situation is somewhat similar to that found in the area of system identification and control, where the tasks of identification and control are typically conflicting [8]. And even when the inputs exhibit sufficient excitation to conduct parameter identification, the resulting solution may still be suboptimal. This is because, not only the outputs predicted 
by the model, but also their derivatives with respect to the inputs, should match the true output values and derivatives, in order for the two-step approach to converge to the actual optimum [9]. A way of dealing with this latter deficiency is to reconcile the objective functions for the identification and optimization problems, e.g., by modifying either the optimization objective (ISOPE approach, [9]) or the identification objective [10]. Note that variants of the ISOPE approach have been proposed recently, where a fixed process model is used and the model parameters need not be updated [11], [12]. In these variants, gradient-correction terms are added to the cost and/or the constraints of the optimization problem so that the computed inputs satisfy the $\mathrm{NCO}$ of the actual plant upon convergence. However, these terms require that the cost and constraint gradients be estimated from the available measurements. For those optimization problems where the solution is mostly determined by active constraints, alternative fixed-model approaches have also been proposed, which update the constraint values without correcting their derivatives [13], [14]. Such constraint-adaptation schemes proceed by simply offsetting the constraints based on their measurements and guarantee a feasible process operation upon convergence.

In this paper, the focus is on explicit optimization of batch processes using a fixed (possibly inaccurate) process model. An extension of constraint-adaptation schemes to dynamic optimization problems that include both path and terminal constraints is proposed. The process model is embedded within a dynamic optimization problem, the constraints of which are updated in a run-to-run fashion by incorporating the available measurements.

The paper is organized as follows. The dynamic optimization problem is formulated in Section II. The run-to-run constraint-adaptation scheme is presented in Section III, and illustrated by a semi-batch reactor example in Section IV. Finally, Section V concludes the paper.

\section{FORMULATION OF THE OPTIMIZATION PROBLEM}

In general, optimizing the operation of a batch process can be formulated as a dynamic optimization problem in the form:

$$
\begin{array}{cl}
\min _{\mathbf{u}(t), t_{f}} & \phi\left(\mathbf{x}\left(t_{f}\right)\right) \\
\text { s.t. } & \dot{\mathbf{x}}=\mathbf{F}(\mathbf{x}, \mathbf{u}), \quad \mathbf{x}(0)=\mathbf{x}_{0} \\
& \mathbf{u}_{\min } \leq \mathbf{u}(t) \leq \mathbf{u}_{\max } \\
& \mathbf{S}(\mathbf{x}, \mathbf{u}) \leq \mathbf{S}_{\max } \\
& \mathbf{T}\left(\mathbf{x}\left(t_{f}\right)\right) \leq \mathbf{T}_{\max }
\end{array}
$$

where $\phi$ is a smooth scalar function representing the terminal cost, $\mathbf{x}$ the $n$-dimensional vector of states with given initial conditions $\mathbf{x}_{0}, \mathbf{u}$ the $m$-dimensional vector of inputs, $\mathbf{S}$ the $\zeta$-dimensional vector of state path constraints, $\mathbf{T}$ the $\tau$-dimensional vector of terminal constraints, $\mathbf{F}$ a smooth vector function describing the system dynamics, and $t_{f}$ the final time. In this formulation, the path constraints on the

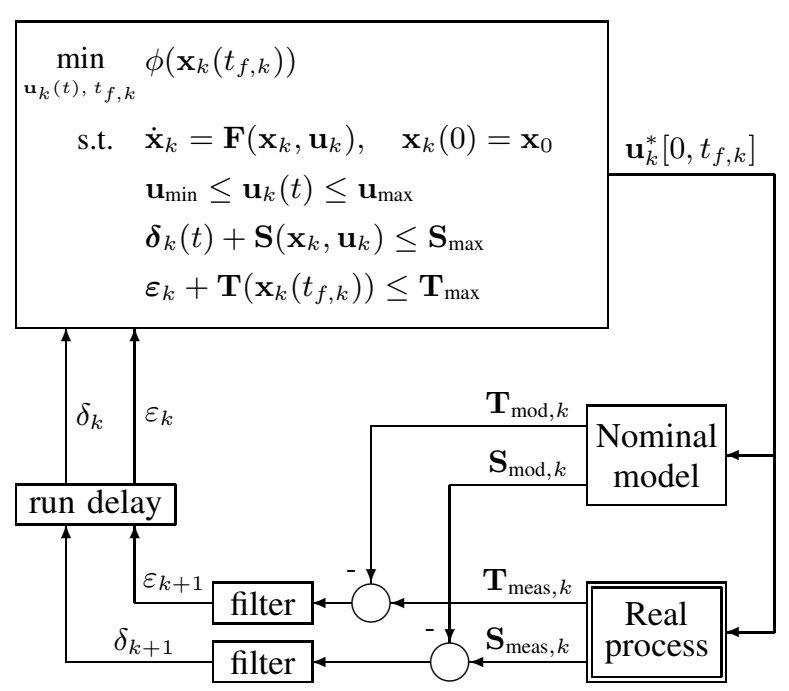

Fig. 1. Run-to-run constraint-adaptation scheme for dynamic optimization.

inputs (or input bounds) are considered separately from the path constraints on the states since only the latter might vary in the presence of uncertainty.

A solution to the dynamic optimization problem (1) typically consists of several arcs over finite time intervals [15]. The optimal inputs are continuous and differentiable within each time interval. The time instants at which the inputs switch from one arc to another are known as the switching times. For a given input, there are two possibilities for each arc: The optimal input is either determined by an active path constraint, i.e., $u=u_{\text {path }}$ (constraint-seeking arc), or it lies inside the feasible region, i.e., $u=u_{\text {sens }}$ (sensitivityseeking arc). Among the constraint-seeking arcs, the input can be at its upper bound $u_{\text {path }}=u_{\max }$, at its lower bound $u_{\text {path }}=u_{\min }$, or determined by an active state path constraint $S\left(\mathbf{x}, u_{\text {path }}\right)=S_{\max }$.

\section{CONSTRAINT-ADAPTATION SCHEME}

The run-to-run MBO structure proposed to address constrained dynamic optimization problems is presented in Fig. 1, where index $k$ indicates the $k^{t h}$ batch. A dynamic optimization problem is solved prior to each batch using the nominal model $\mathbf{F}$ that presents model mismatch with respect to the real process. At the $k^{t h}$ batch the optimal input profiles obtained $\mathbf{u}_{k}^{*}\left[0, t_{f, k}\right]$ are applied to the real process. The same input profiles are applied to the nominal model to obtain by simulation the optimal response $\mathbf{x}_{k}^{*}\left[0, t_{f, k}\right]$. Only the (path and terminal) constraints are adapted between two successive batches, as described subsequently. Because of its nature, the method is particularly well suited to those dynamic optimization problems where meeting the active constraints has a dominant impact on performance.

\section{A. Principles of Constraints Adaptation}

The terminal constraints in the model are adapted in a runto-run fashion to track the terminal constraints measured in 
the real process. This is done by using the additive constraint factors $\varepsilon_{k}$ :

$$
\begin{aligned}
& \varepsilon_{k}+\mathbf{T}_{\text {mod }, k} \leq \mathbf{T}_{\max } \\
& \varepsilon_{k+1}=\left(\mathbf{I}-\mathbf{B}_{\mathrm{T}}\right) \boldsymbol{\varepsilon}_{k}+\mathbf{B}_{\mathrm{T}}\left(\mathbf{T}_{\text {meas }, k}-\mathbf{T}_{\text {mod }, k}\right)
\end{aligned}
$$

where the $\tau$-dimensional vector $\varepsilon_{k}$ is the filtered difference between the terminal constraints measured at the end of the previous run, $\mathbf{T}_{\text {meas, } k-1}$, and the terminal constraints predicted by the model $\mathbf{T}_{\bmod , k-1}:=\mathbf{T}\left(\mathbf{x}_{k-1}^{*}\left(t_{f, k-1}\right)\right) . \mathbf{B}_{\mathrm{T}}$ in (3) is a $\tau \times \tau$ diagonal matrix of filter parameters $b_{\mathrm{T}, i}$ with $i=1, \ldots, \tau$. Each terminal constraint can be filtered individually by setting the parameter $b_{\mathrm{T}, i}\left(0<b_{\mathrm{T}, i} \leq 1\right)$.

The state path constraints in the model are also adapted in a run-to-run fashion to track the state path constraints measured in the real process. This is done by using the additive time-varying constraint factors $\boldsymbol{\delta}_{k}(t)$ :

$$
\begin{aligned}
& \boldsymbol{\delta}_{k}(t)+\mathbf{S}_{\bmod , k}(t) \leq \mathbf{S}_{\max } \\
& \boldsymbol{\delta}_{k+1}(t)=\left(\mathbf{I}-\mathbf{B}_{\mathbf{S}}\right) \boldsymbol{\delta}_{k}(t)+\mathbf{B}_{\mathbf{S}}\left(\mathbf{S}_{\text {meas }, k}(t)-\mathbf{S}_{\text {mod }, k}(t)\right)
\end{aligned}
$$

Likewise, the $\zeta$-dimensional vector $\boldsymbol{\delta}_{k}(t)$ is the filtered difference between the measured state path constraints in the previous run $\mathbf{S}_{\text {meas, } k-1}$, and the values predicted by the model $\mathbf{S}_{\text {mod }, k-1}(t):=\mathbf{S}\left(\mathbf{x}_{k-1}^{*}(t), \mathbf{u}_{k-1}^{*}(t)\right) . \mathbf{B}_{\mathbf{S}}$ in (5) is a $\zeta \times \zeta$ diagonal matrix of filter parameters $b_{\mathrm{S}, j}$ with $j=1, \ldots, \zeta$. Again, each state path constraint can be filtered individually by setting the parameter $b_{\mathrm{S}, j}\left(0<b_{\mathrm{S}, j} \leq 1\right)$. Observe that (4) and (5) are defined for each time instant $t \in\left[0, t_{f}\right]$. In case the final time is not fixed in Problem (1), one option is to use $\boldsymbol{\delta}_{k}(t)=\boldsymbol{\delta}_{k}\left(t_{f, k-1}\right)$ for $t>t_{f, k-1}$.

Analogous to the constraint-adaptation scheme considered in [14] for RTO problems, the exponential filters (3) and (5) are put on the constraint factors and not on the inputs. The rationale behind this choice is that it permits to treat each constraint individually and is thus more flexible.

\section{B. Implementation Aspects}

The dynamic optimization problem in Fig. 1 can be solved with any numerical solution procedure, such as the sequential or the simultaneous method. A tutorial presentation of the most popular solution techniques for dynamic optimization problems can be found in [16].

The application of the constraint-adaptation scheme in Fig. 1 requires that all the state path constraints be measured (or estimated) during the batch. In particular, these measurements can be obtained via off-line analysis whenever on-line measurement is impossible, since the optimization is carried out between successive batches. Likewise, the terminal constraints must be measured (or estimated) at the end of each run, possibly via off-line analysis. Note also that measurement noise as well as small process disturbances can be handled by backing-off from the state path and terminal constraints.

\section{Feasibility Aspects}

The proposed constraint-adaptation scheme utilizes the available measurements to estimate the constraint values, while relying on a fixed process model to estimate the gradients of the cost and constraint functions. In other words, the measurements are used to adapt the inputs along constraint-seeking arcs, whereas the inputs along sensitivityseeking arcs are calculated via the fixed model. The rationale behind this is that meeting path and terminal constraints in dynamic optimization often proves to be more important than meeting the sensitivity part of the NCO; this is confirmed by the variational analysis of the $\mathrm{NCO}$ in the presence of uncertainty [17]. Clearly, the solution obtained through the proposed scheme may be suboptimal, e.g., when the optimal inputs contain sensitivity-seeking arcs. However, for those optimization problems where the solution is mostly determined by the constraints, this scheme pushes the operation towards the constraints of the real process, and nearoptimality is usually achieved within a limited number of runs.

Despite sub-optimality, it is important to ensure that the run-to-run scheme converge towards a feasible operation point, i.e., that the constraints of the real process are satisfied upon convergence. This is established in the following Theorem:

Theorem 1: If the run-to-run constraint-adaptation scheme in Fig. 1 converges, then the path and terminal constraints for the real process are respected.

Proof: Upon convergence, i.e., for $k \rightarrow \infty$, (3) gives $\varepsilon_{\infty}=\mathbf{T}_{\text {meas }, \infty}-\mathbf{T}_{\text {mod, } \infty}$. Inserting this equation into (2) leads to $\mathbf{T}_{\text {meas }, \infty} \leq \mathbf{T}_{\max }$. Similarly, at each time $t \in$ $\left[0, t_{f, \infty}\right]$, (5) gives $\boldsymbol{\delta}_{\infty}(t)=\mathbf{S}_{\text {meas }, \infty}-\mathbf{S}_{\text {mod, } \infty}$, which when inserted into (4) gives $\mathbf{S}_{\text {meas, } \infty} \leq \mathbf{S}_{\max }$.

It should be noted that the adaptation scheme may converge by following an infeasible path, i.e., with violation of the constraints. This highlights the interest of devising an iterative scheme such that, when starting with initial backoffs from the constraints, the iterations follow a feasible path.

It may also happen that the adaptation of the constraints leads to an infeasible optimization problem. A way of tackling this issue is by updating the nominal model, e.g., by adapting some of the model parameters. This does not mean estimating these model parameters accurately, but rather adapting them so as to overcome the infeasibility issue.

A distinction should be made between state path constraints that cannot be physically exceeded and constraints that should not be exceeded. The first kind of constraints typically corresponds to physical limitations due to equipment, while the later corresponds to safety constraints such as an upper temperature bound or an upper bound on the concentration of a hazardous component. In the case where a constraint of the first type reaches its physical bound, the time-varying constraint factor $\boldsymbol{\delta}_{k}(t)$ can no longer be implemented on the real process. On the other hand, safety constraints should not be violated. Hence, a more elaborate scheme can be imagined, wherein the optimal input profiles $\mathbf{u}_{k}^{*}$ given by the $k^{t h}$-optimization are not applied to the real process open-loop because the path constraints can become violated. Instead, the active path constraints in the solution of that optimization problem would be tracked using feedback 
path controllers, as in NCO tracking [4].

\section{Changing Set of Active Constraints}

A desirable property of MBO methods for batch processes lies in their ability to detect changes in the optimal input structure, i.e., to uncover the type and sequence of arcs and the set of active path and terminal constraints that constitute the optimal solution for the actual plant. As mentioned earlier, constraints adaptation alone cannot guarantee that the correct input structure will be found. Much insight on how the method works can be gained by visualizing the situation in the static finite-dimensional case:

$$
\begin{array}{cl}
\min _{\mathbf{u}} & \phi(\mathbf{u}) \\
\text { s.t. } & \mathbf{u}_{\min } \leq \mathbf{u} \\
& \varepsilon+T(\mathbf{u}) \leq T_{\max }
\end{array}
$$

Here, the input $\mathbf{u}$ has two components $u_{1}$ and $u_{2}$, and the constraint $T$ is adapted with the constraint factor $\varepsilon$. Fig. 2a presents the constraint calculated by the model, $T_{\text {mod }}=T_{\max }$, and the location of the constraint for the real process, $T_{\text {meas }}=T_{\max }$. The shadowed area corresponds to the feasible region of the optimization problem using the model with $\varepsilon=0$. Point A represents the optimum calculated with the model in the absence of constraint adaptation, where the active constraints are $u_{2, \min }$ and $T_{\max }$. However, the optimum of the real process is at point $\mathrm{B}$, where the active constraints are $u_{1, \min }$ and $u_{2, \min }$. In this example, depending on the gradient of the cost $\phi_{\mathbf{u}}$ calculated with the model, the adaptation may converge to different sets of active constraints. Fig. 2 b presents the case where, upon adaptation of $T$, the operation converges to the real optimum $\mathrm{B}$. The shadowed area corresponds to the feasible region of the optimization problem using the model with $\varepsilon=\varepsilon_{\mathrm{B}}$, where $\varepsilon_{\mathrm{B}}$ is evaluated at point B. Fig. 2c presents the case where, because of the model mismatch affecting the evaluation of $\phi_{\mathbf{u}}$, the adaptation converges to an incorrect set of active constraints at point $\mathrm{C}$. The shadowed area corresponds to the feasible region given by the model with $\varepsilon=\varepsilon_{\mathrm{C}}$, evaluated at point $\mathrm{C}$. The active constraints in this case are $u_{1, \min }$ and $T_{\max }$.

\section{ILLUSTRATIVE EXAMPLE}

A semi-batch reactor example is considered to illustrate the constraint-adaptation approach.

\section{A. Reaction System}

$$
A+B \longrightarrow C, \quad 2 B \longrightarrow D
$$

The desired product is $C$, while $D$ is undesired. The reactions are exothermic and the operation is made isothermal by using a jacketed reactor. The reactor is initially loaded with $A$, and $B$ is added at a variable feed rate.

\section{B. Model Equations and Parameters}

The dynamic model results from material balance equations:

$$
\begin{aligned}
& \dot{c}_{A}=-r_{1}-\frac{F}{V} c_{A}, \quad c_{A}(0)=c_{A_{0}} \\
& \dot{c}_{B}=-r_{1}-2 r_{2}+\frac{F}{V}\left(c_{B_{\text {in }}}-c_{B}\right), \quad c_{B}(0)=c_{B_{0}} \\
& \dot{V}=F, \quad V(0)=V_{0}
\end{aligned}
$$

with

$$
r_{1}=k_{1} c_{A} c_{B}, \quad r_{2}=k_{2} c_{B}^{2}
$$

Moreover, the following quantities can be reconstructed algebraically from the state variables:

$$
\begin{aligned}
& n_{C}=V_{0} c_{A_{0}}-V c_{A} \\
& n_{D}=\frac{1}{2}\left(V\left(c_{A}-c_{B}\right)+V_{0}\left(c_{B_{0}}-c_{A_{0}}\right)+c_{B_{\text {in }}}\left(V-V_{0}\right)\right) \\
& T_{j}=T_{r}-\frac{V}{U A}\left(\left(-\Delta H_{1}\right) r_{1}+\left(-\Delta H_{2}\right) r_{2}\right)
\end{aligned}
$$

Variables and parameters: $c_{X}$ : concentration of species $X$, $n_{X}$ : number of moles (amount) of species $X, V$ : volume, $r_{i}$ : rate of reaction $i, k_{i}$ : kinetic coefficient of reaction $i$, $F$ : feed rate of $B, \Delta H_{i}$ : enthalpy of reaction $i, T_{r}$ : reactor temperature, $T_{j}$ : cooling jacket temperature, $U$ : heat transfer coefficient, $A$ : reactor heat exchange area.

TABLE I

MODEL PARAMETERS, OPERATING BOUNDS AND INITIAL CONDITIONS

\begin{tabular}{|ccc|ccc|}
\hline$\Delta H_{1}$ & $-8 \times 10^{4}$ & $\mathrm{~J} / \mathrm{mol}$ & $\Delta H_{2}$ & $-10^{5}$ & $\mathrm{~J} / \mathrm{mol}$ \\
$U A$ & $1.25 \times 10^{4}$ & $\mathrm{~J} / \mathrm{min}^{\circ} \mathrm{C}$ & $c_{B_{\text {in }}}$ & 5 & $\mathrm{~mol} / \mathrm{l}$ \\
$T_{r}$ & 30 & ${ }^{\circ} \mathrm{C}$ & $T_{j, \min }$ & 10 & ${ }^{\circ} \mathrm{C}$ \\
$F_{\max }$ & 1 & $1 / \mathrm{min}$ & $F_{\text {min }}$ & 0 & $1 / \mathrm{min}$ \\
$c_{A_{0}}$ & 0.5 & $\mathrm{~mol} / \mathrm{l}$ & $c_{B_{0}}$ & 0 & $\mathrm{~mol} / \mathrm{l}$ \\
$V_{0}$ & 1000 & 1 & $t_{f}$ & 210 & $\mathrm{~min}$ \\
$n_{D, \max }$ & 100 & $\mathrm{~mol}$ & $n_{B, \max }$ & 25 & $\mathrm{~mol}$ \\
\hline
\end{tabular}

TABLE II

KINETIC RATE CONSTANTS FOR NOMINAL MODEL AND SIMULATED REALITY

\begin{tabular}{cccc}
\hline & $k_{1}$ & $k_{2}$ & $k_{1} / k_{2}$ \\
\hline Nominal model & 0.11 & 0.13 & 0.846 \\
Simulated reality A & 0.10 & 0.07 & 1.429 \\
Simulated reality B & 0.09 & 0.18 & 0.5 \\
\hline
\end{tabular}

The model parameters, operating bounds and initial conditions are given in Table I. The reality is simulated by varying the kinetic rate constants $k_{1}$ and $k_{2}$ in the dynamic model. Two scenarios, A and B, are considered. The kinetic rate constants for the nominal model and for the simulated realities $\mathrm{A}$ and $\mathrm{B}$ are presented in Table II. A higher $k_{1} / k_{2}$ ratio implies a better selectivity of product $C$.

\section{Optimization Problem}

The objective is to maximize the amount of product $C$ at final time, $n_{C}\left(t_{f}\right)$, by manipulating the feed rate of $B, F(t)$. A lower bound is imposed on the jacket temperature, and upper bounds are defined for the final amount of byproduct $D$ 
a)

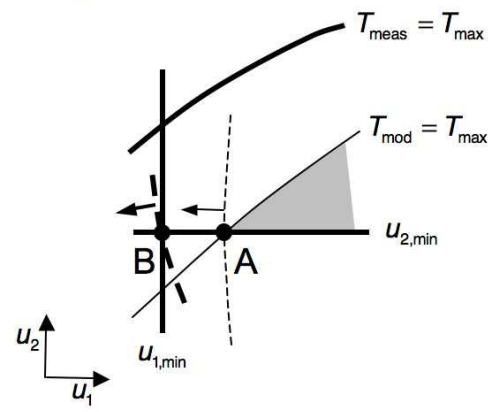

b)

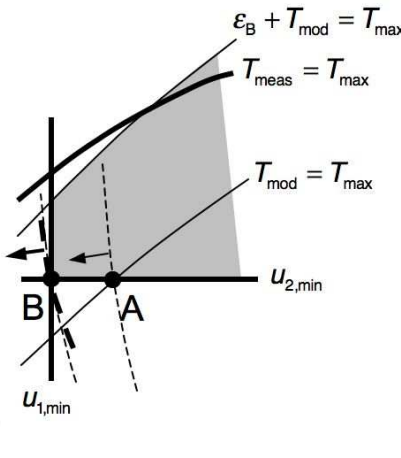

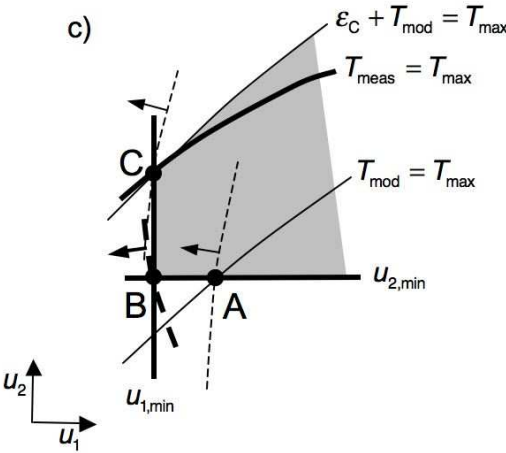

Fig. 2. Sketch of the constraint-adaptation scheme in the finite-dimensional case. Thick solid lines: constraint bounds for the real process. Thin solid lines: constraint bounds evaluated by the model. Thick dashed lines: isoline of $\phi$ corresponding to the real optimum. Thin dashed lines: isolines of $\phi$ evaluated with the model. Arrows: negative of the cost gradient, $-\phi_{\mathbf{u}}$. a) No constraint adaptation. Point A: Nominal model optimum; Point B: Real process optimum. b) Convergence to the real optimum B. c) Convergence to the suboptimal solution C.

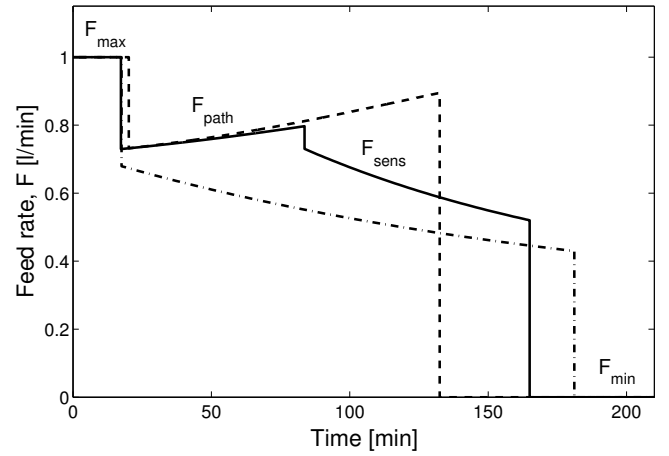

Fig. 3. Optimal input. Solid: nominal model; Dashed: simulated reality A; Dot-dashed: simulated reality B.

and of unreacted $B$ (see Table I). The optimization problem can be formulated mathematically as:

$$
\begin{array}{cl}
\max _{F(t)} & n_{C}\left(t_{f}\right) \\
\text { s.t. } & \text { Model equations } \\
& F_{\min } \leq F(t) \leq F_{\max } \\
& T_{j}(t) \geq T_{j, \min } \\
& n_{D}\left(t_{f}\right) \leq n_{D, \max } \\
& n_{B}\left(t_{f}\right) \leq n_{B, \max }
\end{array}
$$

The optimal input profiles for the nominal model and for the simulated realities A and B are presented in Fig. 3. The corresponding jacket temperature profiles are given in Fig. 4 and the amounts of components $B, C$ and $D$ at final time are given in Table III. Here, the optimization problem was solved numerically using an analytical parameterization approach [16]. The optimal input for the nominal model consists of 4 $\operatorname{arcs} F_{\max }, F_{\text {path }}, F_{\text {sens }}$ and $F_{\min }$, and both terminal constraints on $n_{D}\left(t_{f}\right)$ and $n_{B}\left(t_{f}\right)$ are active. The sensitivity-seeking arc finds a compromise between satisfying the terminal constraint on $n_{D}\left(t_{f}\right)$ and maximizing the production of $C$. Because of the better reaction selectivity, the sensitivityseeking arc is not present in the optimal input for the

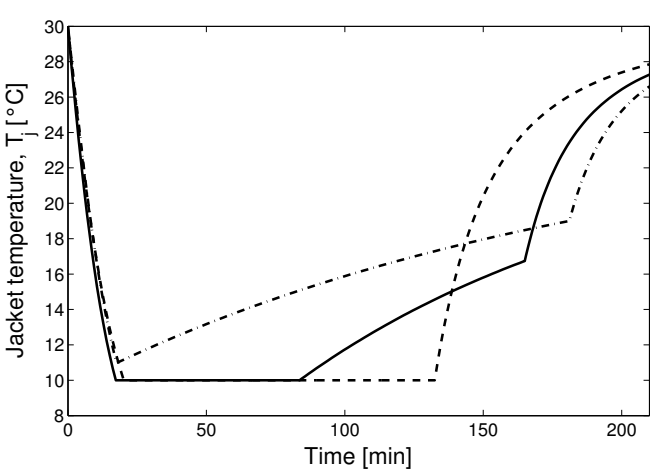

Fig. 4. Optimal jacket temperature profiles. Solid: nominal model; Dashed: simulated reality A; Dot-dashed: simulated reality B.

simulated reality A, and the terminal constraint on $n_{D}\left(t_{f}\right)$ is not active. On the other hand, the simulated reality B has a lower ratio $k_{1} / k_{2}$ and the path constraint is inactive, while both terminal constraints are active.

TABLE III

AMOUNT OF THE VARIOUS SPECIES AT FINAL TIME

\begin{tabular}{cccc}
\hline & $n_{D}\left(t_{f}\right)$ & $n_{B}\left(t_{f}\right)$ & $n_{C}\left(t_{f}\right)$ \\
\hline Nominal model & $100.00^{a}$ & $25.00^{a}$ & 363.59 \\
Simulated reality A & 77.54 & $25.00^{a}$ & 372.83 \\
Simulated reality B & $100.00^{a}$ & $25.00^{a}$ & 301.44 \\
\hline$a$
\end{tabular}

${ }^{a}$ denotes an active constraint.

\section{Adaptation for Simulated Reality A}

Three case studies are presented. In the first one, the adaptation is started with initial constraint factors equal to zero. In the second and third ones, the adaptation is started with constraint factors corresponding to feasible initial operation. The constraint factors $\varepsilon_{1}$ and $\varepsilon_{2}$ correspond to the constraints on $n_{D}\left(t_{f}\right)$ and $n_{B}\left(t_{f}\right)$, respectively.

Case 1) Starting from Infeasible Operation: For the first batch, $\delta_{k=1}\left[0, t_{f}\right]=0$, and $\varepsilon_{1, k=1}=\varepsilon_{2, k=1}=0$. In this case, the method is tested with no measurement noise in order to 
be able to compare the converged solution with the optimal solution of the simulated reality. The filter parameters used are $b_{\mathrm{S}}=1$ and $b_{\mathrm{T}, 1}=b_{\mathrm{T}, 2}=0.7$.

Fig. 5 shows the input calculated for the first, second and tenth runs. The first run corresponds to the nominal optimal input (same as the solid line in Fig. 3). The tenth run is taken as the converged operation. The correct input structure (no sensitivity-seeking arc) and set of active constraints are obtained after a single iteration of the constraint-adaptation scheme. Fig. 6 shows the corresponding jacket temperature profile for the simulated reality. Fig. 7 shows the path constraint factor profiles $\delta_{k}(t)$. Since the constraint on $T_{j}(t)$ is a lower bound, a negative factor $\delta(t)$ corresponds to a back-off of the constraint. The kinetic rate constants for the simulated reality A are smaller than for the nominal model. As a result, the heat released by the reactions is smaller in the real process than for the nominal model and, thus, the constraint factor $\delta(t)$ has a positive value at the beginning of the run. Observe also that the heat released strongly depends on the relative amounts of $A$ and $B$ in the reactor. This is why, when the feed of $B$ is stopped, $\delta(t)$ shows large variations and becomes negative.

The terminal constraint factors used for each input calculation are given in Fig. 8, and the value of the terminal constraints for the simulated reality are given in Fig. 9. The constraint $T_{1}$ on $n_{D}\left(t_{f}\right)$ is satisfied in the first run, while the constraint $T_{2}$ on $n_{B}\left(t_{f}\right)$ is violated. Since for the nominal model both constraints are active with zero constraint factors, the constraint factor for the satisfied constraint, $\varepsilon_{1}$, takes on a negative value, while the constraint factor for the violated constraint, $\varepsilon_{2}$, takes on a positive one.

The amounts of species $D, B$ and $C$ at final time for the converged operation are given in the first row in Table IV, which should be compared to the second row in Table III.

TABLE IV

AMOUNT OF THE VARIOUS SPECIES AT FINAL TIME: CONVERGED VALUES

\begin{tabular}{cccc}
\hline & $n_{D}\left(t_{f}\right)$ & $n_{B}\left(t_{f}\right)$ & $n_{C}\left(t_{f}\right)$ \\
\hline Simulated reality A (Case 1) & 77.54 & $25.00^{a}$ & 372.83 \\
Simulated reality B & $100.00^{a}$ & $25.00^{a}$ & 301.28 \\
\hline${ }^{a}$ denotes an active constraint. & &
\end{tabular}

${ }^{a}$ denotes an active constraint.

Notice that, in this example, the input converges to the exact optimal input in very few runs, although the real kinetics of the reactions are not known precisely. Convergence to the true optimum results from the fact that the optimal input is completely determined by the constraints, i.e., there is no sensitivity-seeking arc in the optimal input of the simulated reality A.

Case 2) Starting from Feasible Operation: Gaussian noises with standard deviations of $0.5 \mathrm{~mol}$ and $0.2 \mathrm{~mol}$ are introduced for the measurement of $n_{D}\left(t_{f}\right)$ and $n_{B}\left(t_{f}\right)$, respectively. In response to this, a back-off of $1 \mathrm{~mol}$ is introduced for the upper bound on $n_{B}\left(t_{f}\right)$, i.e., the method is now applied with $n_{B \text {,max }}=24 \mathrm{~mol}$. When the model uncertainties are parametric with known probability density

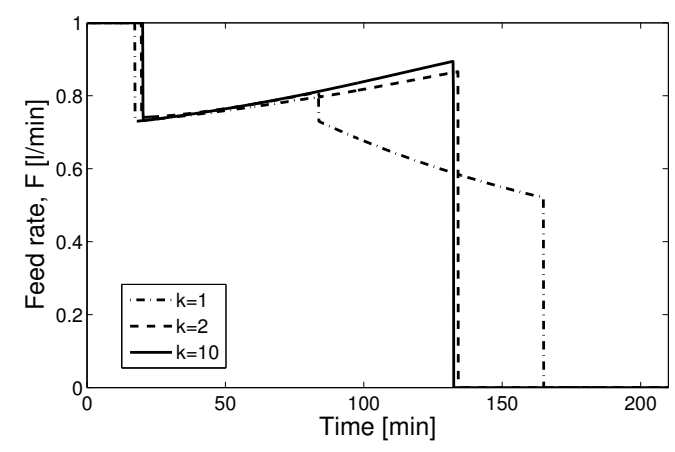

Fig. 5. Simulated reality A - Case 1: Evolution of the input profile with the run index $k$.

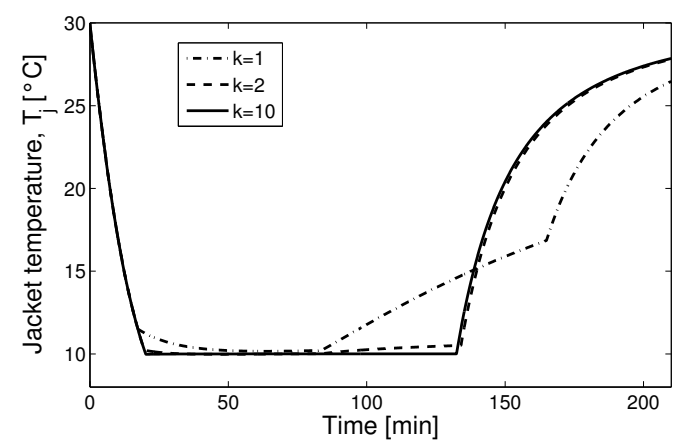

Fig. 6. Simulated reality A - Case 1: Evolution of the jacket temperature profile.

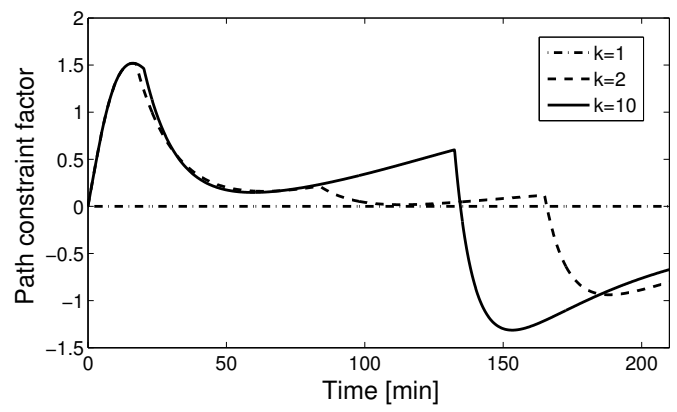

Fig. 7. Simulated reality A - Case 1: Evolution of the path constraint factor $\delta_{k}(t)$.

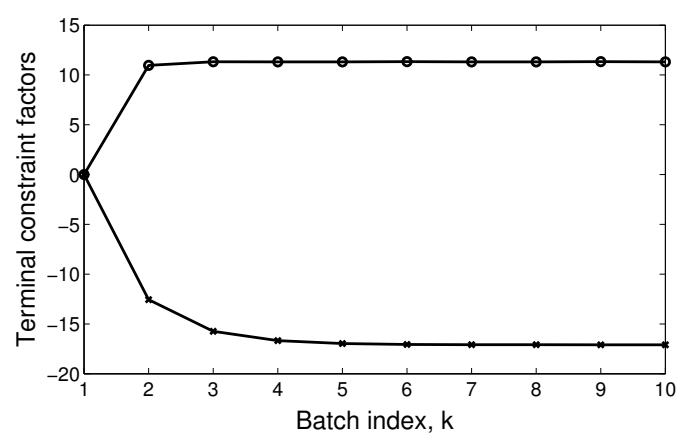

Fig. 8. Simulated reality A - Case 1: Evolution of the terminal constraint factors. Cross: $\varepsilon_{1}$; Circle: $\varepsilon_{2}$ 

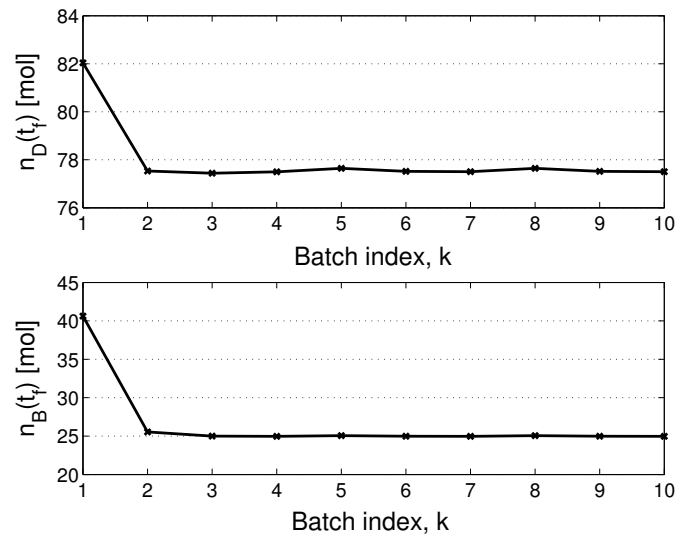

Fig. 9. Simulated reality A - Case 1: Evolution of the constrained variables $n_{D}\left(t_{f}\right)$ and $n_{B}\left(t_{f}\right)$.
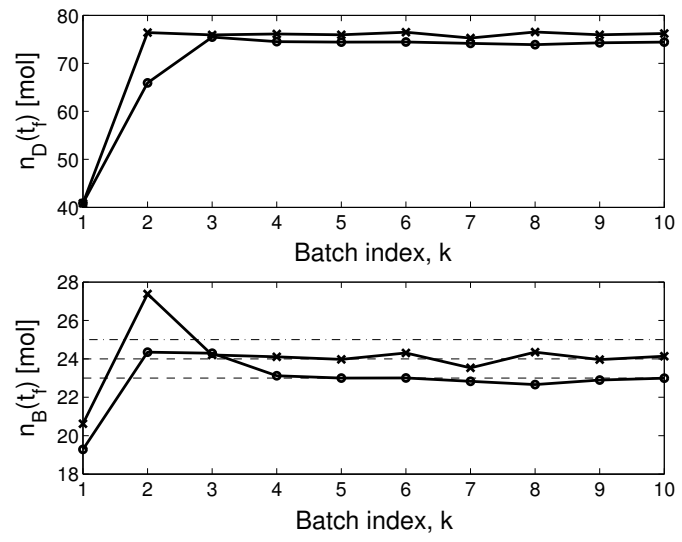

Fig. 10. Simulated reality A. Evolution of the constrained variables $n_{D}\left(t_{f}\right)$ and $n_{B}\left(t_{f}\right)$ when starting from a feasible operating point. Cross: Case 2; Circle: Case 3.

functions or bounds, one can use an iterative procedure to compute back-offs for the constraints that guarantee feasible initial operation (see [4] for details). The following values of the constraint factors: $\delta_{k=1}\left[0, t_{f}\right]=-2, \varepsilon_{1, k=1}=45$, and $\varepsilon_{2, k=1}=11$ are conservative in our example. The filter parameters used are $b_{\mathrm{S}}=1$ and $b_{\mathrm{T}, 1}=b_{\mathrm{T}, 2}=0.7$.

The run-to-run evolution of the measured terminal constraints is presented in Fig. 10. The region where the adaptation is within the noise level is reached in the third batch, i.e, after two runs only. However, although the initial operating point is feasible, the adaptation follows an infeasible path since the constraint on $n_{B}\left(t_{f}\right)$ is violated in the second batch. More filtering, in this case a smaller filter parameter value $b_{\mathrm{T}, 1}$, leads to slower convergence but still results in an infeasible path.

The evolution of the terminal cost $n_{C}\left(t_{f}\right)$ is presented in Fig. 11. About 13\% increase in cost is obtained in 3 batches.

Case 3) Remaining Feasible in Each Batch: The same measurement noise and initial constraint factors as in Case 2 are used. For the operation to remain feasible in any batch, a larger back-off is introduced for the terminal constraint on

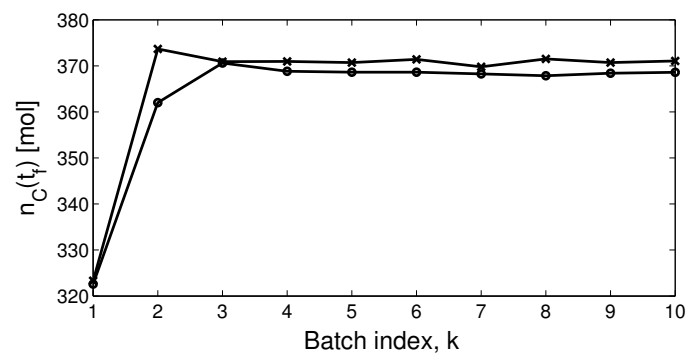

Fig. 11. Simulated reality A. Evolution of the cost $n_{C}\left(t_{f}\right)$. Cross: Case 2; Circle: Case 3.

$n_{B}\left(t_{f}\right)$, i.e. $n_{B, \max }=23 \mathrm{~mol}$, and the filter parameters for the terminal constraints are reduced to $b_{\mathrm{T}, 1}=b_{\mathrm{T}, 2}=0.5$ so as to decrease the overshoot.

The run-to-run evolution of the measured terminal constraints is presented in Fig. 10. The region where the adaptation is within the noise level is reached in the fourth batch. The evolution of the terminal cost $n_{C}\left(t_{f}\right)$ is presented in Fig. 11. The price to pay in order to remain feasible at any iteration is a slower convergence and a loss in performance with respect to Case 2, because of the higher back-off.

Potential improvements include varying the back-offs with the iterations, e.g., starting with larger back-offs and progressively reducing them. Also, the filter parameters could be varied independently from one another, and possibly also with the iterations.

\section{E. Constraints Adaptation for Simulated Reality B}

The constraint-adaptation scheme is applied with $b_{\mathrm{S}}=1$ and $b_{\mathrm{T}, 1}=b_{\mathrm{T}, 2}=1$ with no measurement noise. The initial constraint factors are zero. Fig. 12 shows the input calculated for the first, second and tenth runs. The first run corresponds to the nominal optimal input. The tenth run is taken as the converged operation. Here again, the correct input structure (no state-constraint arc) and set of active terminal constraints are obtained after a single iteration of the constraint-adaptation scheme. The run-to-run evolution of the measured terminal constraints is presented in Fig. 13. The amounts of species $D, B$ and $C$ at final time for the converged operation are given in the second row of Table IV which should be compared to the third row in Table III: The active terminal constraints are the same, and the evaluation of the sensitivity-seeking arc using the incorrect kinetics results in only a marginal optimality loss of $0.05 \%$.

\section{CONCLUSIONS AND FUTURE WORK}

\section{A. Conclusions}

In this paper, a constraint-adaptation scheme has been proposed and studied in the context of batch process optimization. When the optimal solution is determined by the path and terminal constraints of the dynamic optimization problem, a process model, even in the presence of (considerable) model mismatch, can effectively be used for optimization purposes by adapting the constraints of the 


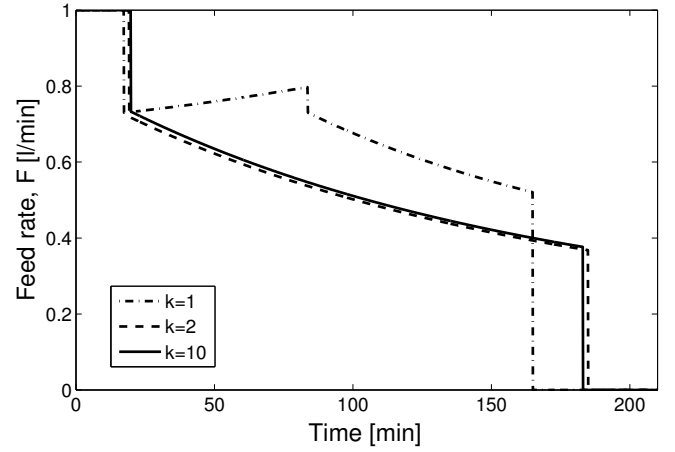

Fig. 12. Simulated reality B. Evolution of input profile with the run index $k$.
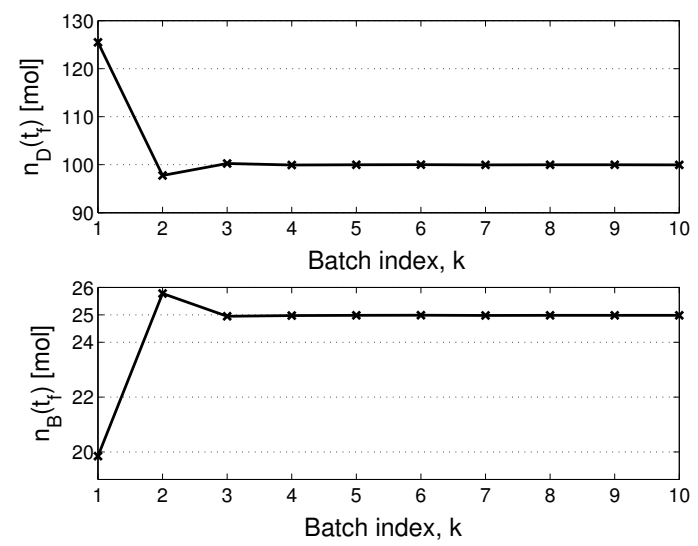

Fig. 13. Simulated reality B. Evolution of the constrained variables $n_{D}\left(t_{f}\right)$ and $n_{B}\left(t_{f}\right)$.

optimization problem. Notice the twist in the approach: Instead of using measurements to update the model in order to predict the accurate values of the constraints, the model remains the same, and the constraints of the optimization problem are updated. This way, model refinement, which requires persistency of excitation in order to uncover the uncertain parameters, is avoided.

When the comparison is done with NCO-tracking techniques that adapt the inputs to track the active path and terminal constraints using feedback laws, the proposed scheme does not require knowledge of the set of active constraints to be tracked. This nice feature is due to the fact that the integrators absorbing the effect of model mismatch are put on the constraints of the optimization problem, and not on the inputs. This represents a major advantage from the implementation viewpoint.

In the example problem used for illustration, the correct input structure is captured for different scenarios, the convergence of the run-to-run iteration is fast, and the presence of a sensitivity-seeking arc in the optimal solution of the real process does not result in an important loss in performance.

\section{B. Future Work}

Future work will address the convergence of the proposed scheme. In particular, the desirable property of converging while enforcing feasible operation at any iteration needs to be investigated. Also, the possibility of using this method along with NCO-tracking methodologies should be considered, since both methods might complement each other well.

\section{REFERENCES}

[1] D. Bonvin, B. Srinivasan, and D. Ruppen, "Dynamic optimization in the batch chemical industry," in Proceedings of the CPC-VI Conference, American Institute of Chemical Engineers, Symposium Series, no. 326 (98), 2002, pp. 255-273.

[2] C. Filippi, J. L. Greffe, J. Bordet, J. Villermaux, J. L. Barnay, B. Ponte, and C. Georgakis, "Tendency modeling of semi-batch reactors for optimization and control," Comp. Chem. Eng., vol. 41, pp. 913-920, 1986.

[3] D. Bonvin, B. Srinivasan, and D. Hunkeler, "Control and optimization of batch processes: Improvement of process operation in the production of specialty chemicals," IEEE Control Systems Magazine, vol. 26, no. 6, pp. 34-45, 2006.

[4] B. Srinivasan, D. Bonvin, E. Visser, and S. Palanki, "Dynamic optimization of batch processes: II. Role of measurements in handling uncertainty," Comp. Chem. Eng., vol. 27, pp. 27-44, 2003.

[5] B. Srinivasan and D. Bonvin, "Real-time optimization of batch processes by tracking the necessary conditions of optimality," Industrial \& Engineering Chemistry Research, vol. 46, no. 2, pp. 492-504, 2007.

[6] E. Zafiriou and J. M. Zhu, "Optimal control of semi-batch processes in the presence of modeling error," in American Control Conference, San Diego, CA, 1990, pp. 1644-1649.

[7] J. Fotopoulos, C. Georgakis, and H. G. Stenger, "Uncertainty issues in the modeling and optimisation of batch reactors with tendency modeling," Chem. Eng. Science, vol. 49, pp. 5533-5548, 1994.

[8] K. J. Åström, "Matching criteria for control and optimization," in European Control Conference, Groningen, The Netherlands, 1993, pp. 248-251.

[9] P. D. Roberts and T. W. C. Williams, "On an algorithm for combined system optimisation and parameter estimation," Automatica, vol. 17, no. 1, pp. 199-209, 1981.

[10] B. Srinivasan and D. Bonvin, "Interplay between identification and optimization in run-to-run optimization schemes," in American Control Conference, Anchorage, Alaska, 2002, pp. 2174-2179.

[11] P. Tatjewski, "Iterative optimizing set-point control - The basic principle redesigned," in 15th IFAC World Congress, Barcelona, Spain, 2002.

[12] W. Gao and S. Engell, "Iterative set-point optimization of batch chromatography," Comp. Chem. Eng., vol. 29, pp. 1401-1409, 2005.

[13] A. Desbiens and A. A. Shook, "IMC-optimization of a direct reduced iron phenomenological simulator," in 4th International Conference on Control and Automation, Montreal, Canada, 2003, pp. 446-450.

[14] A. Marchetti, B. Chachuat, and D. Bonvin, "Real-time optimization of continuous processes via constraints adaptation," in IFAC DYCOPS-8, Cancun, Mexico, 2007.

[15] A. E. Bryson and Y.-C. Ho, Applied Optimal Control. Washington DC: Hemisphere Publishing Corporation, 1975.

[16] B. Srinivasan, S. Palanki, and D. Bonvin, "Dynamic optimization of batch processes: I. Characterization of the nominal solution," Comp. Chem. Eng., vol. 27, pp. 1-26, 2003.

[17] C. Welz, "Measurement-based Optimization of Batch Processes with Terminal Constraints," Ph.D. dissertation, École Polytechnique Fédérale de Lausanne, 2006. 